Evanice Avelino de Souza1,2,3

ODttps://orcid.org/0000-0003-4964-4934

Julio Cesar Barbosa de Lima

Pinto $1,2,3$

Chttps://orcid.org/0000-0002-2066-4408

Felipe Rocha Alves $1,2,3$

Ohttps://orcid.org/0000-0001-9105-2356

\section{Uso do celular antes de dormir: um fator com maior risco para sonolência excessiva em adolescentes de escolas militares}

Cell phone use before bedtime: a risk factor for excessive

sleepiness in adolescents from military schools

D0I: 10.1590/0047-2085000000265

\section{RESUMO}

Objetivo: Investigar a prevalência de sonolência diurna excessiva (SDE) e os fatores associados em adolescentes de escolas militares. Métodos: Participaram 466 adolescentes do ensino médio (15 a 17 anos) de ambos os sexos (230 homens). Os participantes foram entrevistados sobre as características sociodemográficas e sobre a duração de sono (semana e final de semana), adotando-se valores $<8$ horas de sono como tempo insuficiente de sono. A SDE foi avaliada com a escala de sonolência de Epworth, na qual os adolescentes que obtivessem escores $\geq 10$ apresentavam SDE. Resultados: A prevalência geral de SDE foi de $34,1 \%$. A média de horas de sono foi de $6,9 \mathrm{~h}( \pm 1,85)$ em dias da semana e de $8,5 \mathrm{~h}( \pm 1,96)$ nos fins de semana. Identificou-se que $60 \%$ dos adolescentes apresentaram baixa duração do sono semanal ( $p=0,05)$. Observaram-se, no modelo final de regressão, maiores riscos de SDE nos indivíduos com baixa duração de sono (OR: 1,55; IC de 95\%: 1,04-2,31) e que utilizavam o celular antes de dormir (OR: 4,30; IC de 95\%: 2,00-9,23). Conclusão: A SDE foi fortemente associada ao uso de celular antes de dormir. Outros fatores associados são sono insuficiente e estudar em tempo integral. Medidas educacionais, administrativas e de saúde são necessárias para melhorar o sono em adolescentes.

\section{PALAVRAS-CHAVE}

Higiene do sono, estudantes, saúde do estudante.

\begin{abstract}
Objective: To investigate the prevalence of excessive daytime sleepiness (EDS) and associated factors in adolescents attending military schools. Methods: 466 high school students (15-17 years old) of both sexes (230 men) participated. Participants were interviewed about sociodemographic characteristics and sleep duration (week and weekend), adopting values $<8$ hours of sleep as insufficient sleep time. EDS was assessed with the Epworth Sleepiness Scale; where students with scores $\geq 10$ had EDS. Results: The overall prevalence of EDS was $34.1 \%$. The mean for sleep hours was $6.9 \mathrm{~h}( \pm 1,85)$ on weekdays and $8.5 \mathrm{~h}( \pm 1.96)$ on weekends. It was identified that $60 \%$ of the adolescents presented short weekly sleep duration $(p=0.05)$. In the final regression model, higher EDS risks were observed in those subjects that had a short sleep duration (OR: 1.55; 95\% Cl: 1.04-2.31) and those that used the cell phone before bedtime (OR: 4.30; 95\% Cl: 2.00-9.23). Conclusion: EDS was strongly associated with cell phone use before bedtime. Other associated factors are insufficient sleep and studying full-time. Educational, administrative and health measures are needed to improve sleep in adolescents.
\end{abstract}

KEYWORDS

Sleep hygiene, students, student health.

Received in: Feb/23/2020. Approved in: Mar/25/2020

1 Universidade Federal do Ceará (UFC), Faculdade de Medicina, Programa de Pós-graduação em Ciências Médicas, Fortaleza, CE, Brasil.

2 Universidade Federal do Ceará (UFC), Laboratório do Sono e Ritmos Biológicos, Fortaleza, CE, Brasil.

3 Faculdade Terra Nordeste, Curso de Educação Física, Caucaia, CE, Brasil.

Address for correspondence: Evanice Avelino de Sousa. Universidade Federal do Ceará (UFC), Faculdade de Medicina. Rua Monsenhor Furtado, s/n, Rodolfo Teófilo -60441-750 - Fortaleza, CE, Brasil.E-mail: profeas@gmail.com 


\section{INTRODUÇÃO}

O sono é essencial para a maturação física, intelectual e emocional de crianças e adolescentes e, consequentemente, para o desenvolvimento da saúde. Bons hábitos relacionados ao ato de dormir são fundamentais para a promoção de um sono saudável', no entanto as dificuldades em manter e estabelecer os bons hábitos de sono constituem uma crescente preocupação apresentada pelos profissionais de saúde².

Durante a adolescência, é possível observar importantes mudanças na expressão do ciclo sono-vigília, que incluem um atraso na fase de sono, caracterizado por horários de dormir e acordar mais tardios ${ }^{3}$. Esse atraso de fase é exacerbado pelo aumento da interação social noturna e está em contraste com os horários de início das aulas em período matutino, o que contribui significativamente para a redução das horas de sono ${ }^{4}$. Destaca-se, ainda, que, com o avanço da adolescência, existe a possibilidade de que o indivíduo determine seus próprios horários de sono 5 .

A consequência mais direta do sono de má qualidade e/ou insuficiente é a sonolência diurna excessiva (SDE), que afeta o desempenho acadêmico, reduzindo a atenção, a consolidação da memória e a aprendizagem ${ }^{6}$. A SDE é reconhecida como problema mundial de saúde pública, afetando 27,6\% dos adolescentes da zona rural da China 7 , 18\% na Noruega ${ }^{8}$, 18,7\% na Coreia ${ }^{9}$ e 13\% a 24\% no sudeste da Finlândia ${ }^{10}$.

É amplamente aceito que a exposição a fatores de risco comportamentais, incluindo hábitos irregulares de sono, se inicia na adolescência e que esses comportamentos podem ser transferidos para a vida adulta"1. Nesse contexto, o uso de aparelhos eletrônicos vem crescendo cada vez mais entre adolescentes ${ }^{12-14}$, principalmente no momento em que eles vão dormir ${ }^{15,16}$, pois tendem a ficar deitados e, assim, a permanecer utilizando os aplicativos de conversação ou redes sociais ${ }^{17}$. Dessa maneira, a problemática da baixa duração do sono, já diagnosticada nesse período da vida, pode ser agravada com o uso desses aparelhos próximo ao horário de dormir.

O horário de início das aulas é outro fator que desempenha um papel significativo. Estudos anteriores realizados com adolescentes indicaram que atrasar o horário de início da escola pode resultar em benefícios sustentados na duração do sono, no estado de alerta diurno e nos problemas mentais ${ }^{18,19}$. No Brasil, o formato de operação da escola tem sido tradicionalmente organizado em três turnos, com turnos da manhã e da tarde, e para adolescentes de 15 anos ou mais, particularmente aqueles que trabalham durante o dia, geralmente é possível frequentar a escola à noite ${ }^{20,21}$. Mais recentemente, várias escolas adotaram o sistema de tempo integral, com o objetivo explícito de aumentar a exposição acadêmica, os programas extracurriculares e a qualidade da educação ${ }^{22}$.

Apesar do crescente interesse em investigar o sono em adolescentes, os estudos latino-americanos que investigam a SDE na adolescência como desfecho primário ainda são escassos ${ }^{23}$; até onde sabemos, até momento nenhum estudo brasileiro foi realizado em escolas militares, que possuem uma realidade bem diferente da maior parte do sistema de ensino brasileiro, especialmente o público. Primeiramente, porque são em menor número em relação ao total de estabelecimentos de ensino, além de pré-selecionaram por meio de testes classificatórios, por causa do pequeno número de vagas em relação à demanda, esses colégios atraem um perfil de estudantes que, em sua maioria, buscam ingressar na profissão militar ${ }^{24}$.

A busca de um aprofundamento a respeito da SDE e os fatores associados pode contribuir para a implementação de propostas coerentes de intervenção. Assim, o objetivo do presente estudo foi investigar a prevalência de SDE e os fatores associados em adolescentes de escolas militares de uma cidade do nordeste do Brasil.

\section{MÉTODOS}

Estudo transversal realizado na cidade de Fortaleza, Ceará, nordeste do Brasil. Em 2012, a cidade de Fortaleza era a quinta capital mais populosa do Brasil, com aproximadamente 2.591.114 milhões de habitantes e Índice de Desenvolvimento Humano Municipal de 0,732. Na referida cidade, existem três escolas militares: o Colégio da Polícia Militar do Ceará General Edgard Facó (CPMGEF), o Colégio Militar do Corpo de Bombeiros do Estado do Ceará (CBM) e o Colégio Militar de Fortaleza (CMF). Entretanto, não se obteve autorização do CMF para a realização da pesquisa, participando somente os escolares das duas outras já referidas escolas.

De acordo com a secretaria das escolas envolvidas, no ano de 2017 existiam 878 adolescentes regularmente matriculados no ensino médio, distribuídos da seguinte forma: Colégio Militar do Corpo de Bombeiros $(n=373)$ e Colégio da Polícia Militar General Edgard Facó $(n=505)$. A população total, a amostra final e os critérios de exclusão da amostra estão ilustrados na figura 1. Todos os participantes incluídos nesta pesquisa foram devidamente esclarecidos dos riscos e benefícios da pesquisa e concordaram em participar assinando o Termo de Consentimento Livre e Esclarecido devidamente aprovado pelo Comitê de Ética e Pesquisa da Faculdade Terra Nordeste (protocolo no 2.933.313).

Os dados foram coletados no período de abril a outubro de 2017. Os questionários foram aplicados pelos pesquisadores e preenchidos pelos estudantes em sala de aula, sem limite de tempo. Estudantes que faltaram à escola no dia da administração não foram incluídos no estudo. As avaliações foram realizadas nos turnos da manhã e tarde.

\section{Variáveis independentes}

As características sociodemográficas incluíram: sexo, idade, série e turno de estudo (manhã, tarde e integral). Fatores 


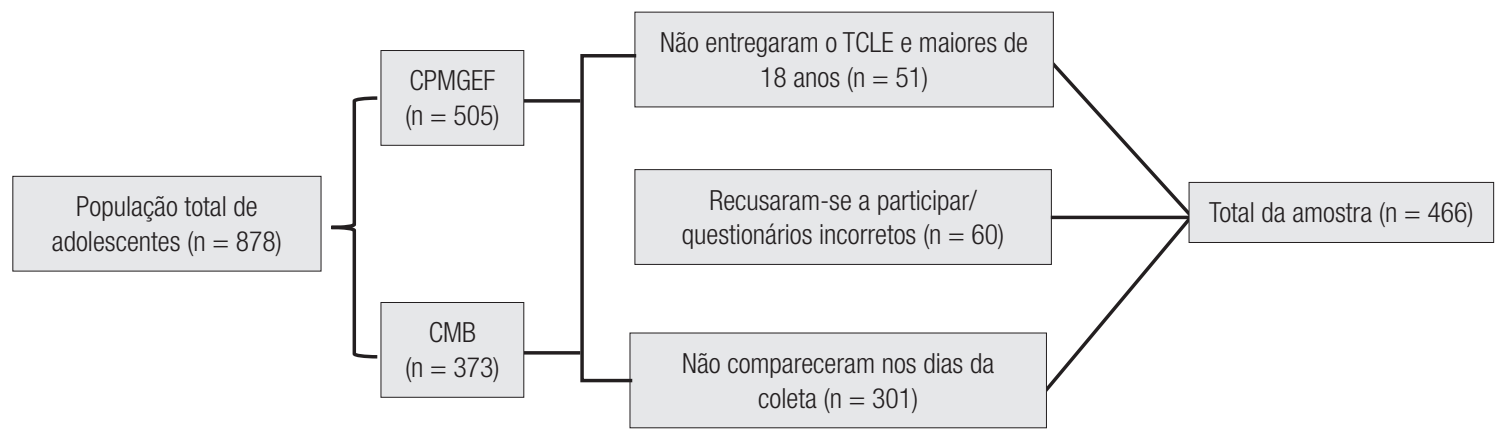

Figura 1. População e amostra de adolescentes das escolas militares da cidade de Fortaleza.

comportamentais incluídos: atividade física (avaliada pela pergunta: "Você pratica atividade física regularmente? Sim ou não); uso de telefone celular antes de dormir (avaliado pela pergunta: "Você usa celular antes de dormir? Sim ou não).

A duração do sono foi medida pela quantidade de tempo que os adolescentes dormem por noite. Foram considerados dias com aula (domingo para segunda-feira, segunda para terça-feira, terça para quarta-feira, quarta para quinta-feira e quinta para sexta-feira) e dias sem aula (sexta-feira para sábado e sábado para domingo). Considerou-se que os adolescentes com menos de 8 horas de sono por noite tinham duração insuficiente de sono, segundo recomendações ${ }^{25,26}$.

\section{Variável dependente}

A sonolência diurna foi avaliada pela versão brasileira da escala de sonolência de Epworth ${ }^{27}$, adaptada para crianças e adolescentes ${ }^{28}$, utilizada anteriormente em estudo com adolescentes brasileiros ${ }^{21}$. É composta por oito questões sobre a probabilidade de cochilar ou adormecer em oito situações hipotéticas, quantificadas como zero (sem chance), 1 (chance pequena), 2 (chance moderada) ou 3 (chance alta). O escore final varia de zero a 24 pontos e os escores $\geq 10$ são considerados indicativos de SDE27,28.

\section{Análise estatística}

A estatística descritiva foi utilizada por meio da média, desvio-padrão e porcentagem. O teste do qui-quadrado de heterogeneidade de Pearson foi usado para verificar a associação significativa entre SDE com sexo, idade, série, turno de estudo, participação nas aulas de educação física, prática de atividade física, uso de celular antes de dormir e duração do sono em dias da semana e aos finais de semana. Utilizou-se ainda o teste do qui-quadrado para comparar a proporção de adolescentes com baixa duração do sono na semana e aos fins de semana.

As variáveis que apresentaram diferença significativa pelo teste do qui-quadrado (turno de estudo, uso de celular antes de dormir e horas de sono semanal) foram incluídas em um modelo de regressão logística univariada e posteriormente inseridas no modelo multivariado.
Nesse modelo, todas as variáveis que apresentaram valor de $p<0,25$ na regressão logística univariada foram adicionadas ao modelo múltiplo. Neste último, foi considerada estatisticamente significante a relação de variáveis com $p<$ 0,05 . Dessa forma, foram calculados os odds ratio (OR) com intervalos de confiança (IC) de 95\%

As análises foram realizadas pelo SPSS, IBM (v. 20.0 for Windows, Chicago, IL, USA), com nível de significância $p<0,05$.

\section{RESULTADOS}

Os dados descritivos dos 466 escolares relacionados à sonolência são apresentados na tabela 1, na qual se identificou que estudar no período integral $(47,1 \%)$, dormir menos de 8 horas durante a semana $(37,3 \%)$ e utilizar celular antes de dormir (36,6\%) esteve relacionado a SDE.

A média de horas de sono foi de 6,9 horas $( \pm 1,85) \mathrm{em}$ dias da semana e de 8,5 horas $( \pm 1,96)$ nos fins de semana. Observou-se que $34,1 \%$ dos adolescentes apresentam SDE. Verificou-se diferença estatística $(p=0,012)$ em relação às horas de sono semanais e aos fins de semana (Figura 2).

$\mathrm{Na}$ análise univariada, verificou-se que estudar em período integral (OR: 2,14; IC de 95\%: 1,34-3,43), usar o celular antes de dormir (OR: 4,68; IC de 95\%: 2,20-9,93) e ter menos de 8 horas semanais de sono (OR: 1,67; IC de 95\%: 1,15-2,43) são fatores de maiores riscos para SDE.

Considerando as variáveis significativas na análise univariada, identificamos no modelo final da regressão que usar o celular antes de dormir e ter menos de 8 horas semanais de sono são os fatores de risco para SDE na amostra investigada (Figura 3).

\section{DISCUSSÃO}

A prevalência da SDE relacionada ao uso de celular, turno de estudo em período integral e baixa duração do sono foram os principais resultados identificados neste estudo, que até o presente momento é a primeira pesquisa realizada sobre sono em adolescentes do ensino militar da cidade de Fortaleza. 
Tabela 1. Características sociodemográficas relacionadas ao grau de SDE dos adolescentes das escolas militares (Fortaleza, CE, 2018)

\begin{tabular}{|c|c|c|c|}
\hline \multirow{2}{*}{$\begin{array}{l}\text { Variáveis sociodemográficas } \\
(\mathrm{n} / \%)\end{array}$} & \multicolumn{3}{|c|}{ Total $(n=466)$} \\
\hline & $\begin{array}{l}\text { Sem SDE } \\
(n=307)\end{array}$ & $\begin{array}{c}\text { SDE } \\
(n=159)\end{array}$ & $\mathbf{p}^{\star}$ \\
\hline \multicolumn{4}{|l|}{ Sexo } \\
\hline Masculino & $162(70,4)$ & $68(29,6)$ & \multirow[t]{2}{*}{0,026} \\
\hline Feminino & $145(61,4)$ & $91(38,6)$ & \\
\hline \multicolumn{4}{|l|}{ Idade (anos) } \\
\hline 15 & $90(71,4)$ & $36(28,6)$ & \multirow{3}{*}{0,189} \\
\hline 16 & $123(66,1)$ & $63(33,9)$ & \\
\hline 17 & $94(61,0)$ & $60(39,0)$ & \\
\hline \multicolumn{4}{|l|}{ Série (ensino médio) } \\
\hline $1^{\circ}$ ano & $144(67,0)$ & $71(33,0)$ & \multirow{3}{*}{0,062} \\
\hline $2^{\circ}$ ano & $129(69,0)$ & $58(31,0)$ & \\
\hline $3^{\circ}$ ano & $34(53,1)$ & $30(46,9)$ & \\
\hline \multicolumn{4}{|l|}{ Turno de estudo } \\
\hline Manhã & $83(61,5)$ & $52(38,5)$ & \multirow{3}{*}{0,006} \\
\hline Tarde & $187(71,6)$ & $74(28,4)$ & \\
\hline Integral & $37(52,9)$ & $33(47,1)$ & \\
\hline \multicolumn{4}{|l|}{ Prática de atividade física } \\
\hline Sim & $192(66,0)$ & $99(34,0)$ & \multirow[b]{2}{*}{0,351} \\
\hline Não & $100(63,7)$ & $57(36,3)$ & \\
\hline \multicolumn{4}{|l|}{ Usa celular antes de dormir } \\
\hline Sim & $256(63,4)$ & $148(36,6)$ & \multirow[b]{2}{*}{0,001} \\
\hline Não & $38(88,4)$ & $5(11,6)$ & \\
\hline \multicolumn{4}{|l|}{ Horas de sono semanal } \\
\hline$<8 \mathrm{~h}$ & $175(62,7)$ & $104(37,3)$ & \multirow[b]{2}{*}{0,048} \\
\hline$\geq 8 \mathrm{~h}$ & $132(70,6)$ & $55(29,4)$ & \\
\hline \multicolumn{4}{|l|}{ Horas de sono (final de semana) } \\
\hline$<8 \mathrm{~h}$ & $57(62,0)$ & $35(38,0)$ & \multirow[b]{2}{*}{0,222} \\
\hline$\geq 8 \mathrm{~h}$ & $250(66,8)$ & $124(33,2)$ & \\
\hline
\end{tabular}

SDE: sonolência diurna excessiva.

* Teste do qui-quadrado de heterogeneidade de Pearson

Tabela 2. Modelo de regressão linear univariada dos fatores associados com a SDE em adolescentes das escolas militares (Fortaleza, CE, 2018)

\begin{tabular}{lccc}
\hline \multirow{2}{*}{ Variáveis } & \multicolumn{2}{c}{ SDE (34,1\%) } & \multirow{2}{*}{$\mathbf{p}^{*}$} \\
\cline { 2 - 3 } & $\mathbf{O R}$ & $\mathbf{I C} \mathbf{( 9 5 \% )}$ & \\
\hline $\begin{array}{l}\text { Turno de estudo } \\
\quad \text { Manhã }\end{array}$ & 1,19 & $(0,80-1,76)$ & 0,320 \\
$\quad$ Tarde & 1 & - & \\
$\quad$ Integral & 2,14 & $(1,34-3,43)$ & 0,003 \\
Uso do celular & & & \\
$\quad \begin{array}{l}\text { Sim } \\
\text { Não }\end{array}$ & 4,68 & $(2,20-9,93)$ & 0,001 \\
Horas de sono semanal & 1 & - & \\
$\quad<8$ h & & & \\
$\geq 8 \mathrm{~h}$ & 1,67 & $(1,15-2,43)$ & 0,001 \\
\hline
\end{tabular}

SDE: sonolência diurna excessiva; OR: odds ratio; IC: intervalo de confiança.

*Análises univariadas com p > 0,25 não foram incluídas na Regressão Logística Multivariada

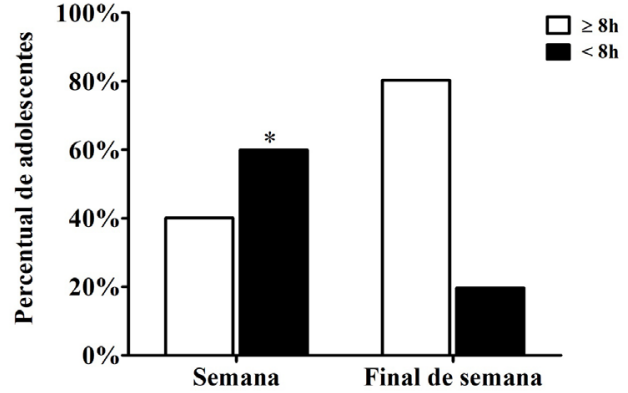

* Diferença significativa $p<0,05$.

Figura 2. Frequência de adolescentes com alta e baixa duração do sono na semana e aos FDS das escolas militares de Fortaleza.

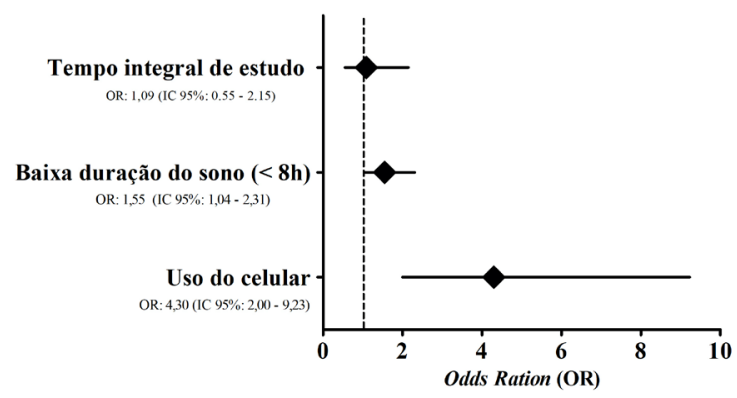

IC: intervalo de confiança.

Figura 3. Fatores associados à sonolência diurna excessiva em adolescentes das escolas militares (Fortaleza, CE, 2018).

Identificou-se, no presente estudo, uma média 6,9 horas de sono em dias com aula, sendo inferior aos resultados encontrados em estudos nacionais ${ }^{29-31}$ e internacionais ${ }^{32-35}$, principalmente comparando-se a Suécia e Estônia, onde 76\% e $68 \%$ dos adolescentes, respectivamente, reportaram dormir entre 8 e 9 horas nos dias com aula ${ }^{36}$. Como verificado, não existe uma consistência do padrão de duração do sono entre diferentes países; possivelmente as diferenças culturais, sociais e climáticas, bem como atividades obrigatórias dos adolescentes, podem acabar influenciando na quantidade de sono. Contudo, os países europeus apresentaram uma maior duração de sono comparados ao Brasil, sugerindo que as políticas públicas desses países possivelmente se preocupam com uma duração suficiente de sono.

Esse resultado pode estar relacionado ao contexto acadêmico vivenciado pelos adolescentes do presente estudo. Além de obedecer aos princípios da educação básica brasileira, as práticas didático-pedagógicas nas escolas militares subordinam-se às normas e prescrições do sistema de ensino militar, baseado na disciplina e rigidez ${ }^{24}$. Sendo assim, a baixa duração do sono registrada em dias da semana nos adolescentes entrevistados pode estar relacionada ao estresse acadêmico experimentado por esses estudantes, que passam a maior parte do ano letivo final preparando-se para 
exames internos e/ou ingresso na universidade ${ }^{24}$. Como o sono é essencial para a aprendizagem e a consolidação da memória, sugere-se obter informações sobre higiene do sono em escolas militares.

Paralelamente à baixa duração de sono, já relatada, verificou-se uma prevalência de SDE relacionada ao turno de estudo, e os adolescentes que frequentavam a escola em tempo integral apresentaram maiores chances em ter SDE. No Brasil, o início das aulas começa por volta das 7 horas da manhã para os estudantes que frequentam os turnos da manhã ou período integral. Na adolescência, há um atraso fisiológico na fase de sono, com uma tendência natural de ir dormir e acordar tarde 3 . Evidências de estudos anteriores mostram que o início precoce das atividades escolares contribui negativamente para o tempo total de sono entre adolescentes ${ }^{4,37,38}$. O atraso no horário de início das aulas tem sido proposto como estratégia para reduzir a restrição de sono em adolescentes ${ }^{39}$. June et al. ${ }^{18}$ avaliaram o impacto de um atraso de 45 minutos nos horários de início das aulas sobre o sono, a sonolência e o bem-estar de adolescentes de Singapura e observaram melhora na duração total do sono a curto e longo prazo, bem-estar e redução da SDE. Resultados semelhantes foram relatados em um estudo recente dos EUA, no qual estudantes que frequentavam escolas que começaram 37 minutos depois tiveram uma média de $17 \mathrm{mi}-$ nutos a mais de sono e menos sonolência comparados com aqueles em escolas de início precoce ${ }^{19}$. Bacelar et al. ${ }^{40}$ registram uma média de 6,7 horas de sono em dias de semana em adolescentes brasileiros. Em conclusão, os autores propõem que as aulas não tenham início antes das 8 h30 para adolescentes brasileiros que estão cursando o ensino médio ${ }^{40}$. Porém, trata-se de uma medida de difícil implementação, considerando os sistemas educacionais e a necessidade de alteração não somente dos horários dos estudantes, mas de toda a comunidade escolar, inclusive dos pais ${ }^{3,21}$.

Entre as variáveis investigadas, o uso do celular antes de dormir apresentou-se como maior risco para SDE. Um estudo realizado na região central da suíça com adolescentes entre 12 e 17 anos encontrou uma associação do uso de celular antes de dormir com cansaço, exaustão rápida, dor de cabeça e mal-estar físico ${ }^{41}$. Na Turquia, entre os 2.021 adolescentes que usavam celular, foi identificada uma relação do número de chamadas por dia, do número total de mensagens de texto por dia e do status do telefone celular durante a noite com dor de cabeça, fadiga e distúrbios do sono, além de aquecimento da orelha e rubor como sintomas locais ${ }^{16}$. Portanto, são importantes programas de conscientização quanto ao uso do celular por adolescentes, não somente antes de dormir ${ }^{8}$, mas de forma geral, por possíveis agravos ao bem-estar físico mental.

É importante considerar algumas limitações deste estudo. Primeiro, deve-se ter em mente que, devido ao delineamento transversal deste estudo, não é possível estabelecer relações de causa e efeito entre as variáveis de exposição e desfecho. Porém, estudos transversais representam a etapa inicial do processo de monitoramento em saúde e podem sugerir elementos importantes para desenhos subsequentes e medidas para mudanças. Segundo, informações sobre duração do sono foram obtidas por autorrelato. Contudo, as avaliações de autorrelato do sono demonstraram ser medidas válidas em comparação com os métodos objetivos de avaliação do sono ${ }^{35}$. A pergunta sobre atividade física e uso de celular antes de dormir foram realizadas sem quantificação. Especialmente sobre o uso de celular antes de dormir, sugere-se que futuros estudos investiguem o tempo de uso desses aparelhos eletrônicos próximo ao horário de dormir, pois essa informação seria extremamente importante para a compreensão dos resultados. Apesar disso, este estudo destaca-se por ser um dos primeiros realizados no Brasil investigando a relação da sonolência com o uso de celulares antes de dormir. Adicionalmente, a sonolência diurna não foi mensurada objetivamente. Embora o teste de latência múltipla do sono, que quantifique a capacidade de adormecer em várias oportunidades repetidas, seja geralmente considerado a medida objetiva padrão da $\mathrm{SDE}^{36}$, seu uso na presente amostra não seria prático. Por outro lado, a Epworth Sleepiness Scale é a medida subjetiva mais comumente usada no contexto clínico e na investigação epidemiológica ${ }^{39}$. Finalmente, é importante considerar que, devido a supostas restrições de tempo alegadas pelas escolas participantes, a amostra final do estudo constituiu em apenas 57\% do público-alvo do estudo.

\section{CONCLUSÃO}

Os adolescentes do presente estudo apresentam baixa duração do sono em dias com aula. A SDE apresentou forte associação com o uso de celular antes de dormir. Outro resultado importante foi a relação existente entre a SDE e a baixa duração do sono e o turno de estudo. Como o sono é essencial para a aprendizagem, é interessante um estímulo ao desenvolvimento de programas educacionais sobre o sono como programas de políticas públicas no ambiente escolar, com extensão para os pais e comunidade, para indicar a mudança de comportamentos e até mesmo a mudança do turno de estudo, influenciando positivamente o desempenho acadêmico e emocional dos estudantes, bem como diminuição da SDE, em vista da carência de estudos randomizados de intervenção para crianças e adolescentes brasileiros.

\section{CONTRIBUIÇÕES INDIVIDUAIS}

Todos os autores contribuíram significativamente para o manuscrito, da seguinte maneira: Evanice Avelino de Souza 
participou de todas as etapas do estudo, da definição e delimitação da coleta, da análise dos dados e da escrita do artigo. Julio Cesar Barbosa de Lima Pinto participou da elaboração das ilustrações, da interpretação dos resultados e da discussão. Felipe Rocha Alves participou da definição do estudo, auxiliou toda a coleta de dados e contribuiu na escrita do artigo.

\section{CONFLITO DE INTERESSES}

Os autores declaram não haver conflito de interesses.

\section{REFERÊNCIAS}

1. Sluggett $L$, Wagner $L S$, Harris RL. Sleep duration and obesity in children and adolescents. Can J Diabetes. 2019;43(2):146-52.

2. Hansen SL, Capener D, Daly C. Adolescent sleepiness: causes and consequences. Pediatr Ann. 2017;46(9):e340-4.

3. Felden ÉPG, Filipin D, Barbosa DG, Andrade RD, Meyer C, Louzada FM. Factors associated with short sleep duration in adolescents. Rev Paul Pediatr. 2016;34(1):64-70.

4. Al-Hazzaa HM, Musaiger A0, Abahussain NA, Al-Sobayel HI, Qahwaji DM. Lifestyle correlates of self-reported sleep duration among Saudi adolescents: a multicentre schoolbased cross-sectional study. Child Care Health Dev. 2014;40(4):533-42.

5. Gibson ES, Powles AC, Thabane L, O'Brien S, Molnar DS, Trajanovic N, et al. "Sleepiness" is serious in adolescence: Two surveys of 3235 Canadian students. BMC Public Health. 2006;6:116.

6. Moore Melisa, Meltzer Lisa J. The sleepy adolescent: causes and consequences of sleepiness in teens. Paediatr Respir Rev. 2008;9(2):114-20; quiz 120-1.

7. Luo C, Zhang J, Chen W, Lu W, Pan J. Course, risk factors, and mental health outcomes of excessive daytime sleepiness in rural Chinese adolescents: a one-year prospective study. J Affect Disord. 2018;231:15-20.

8. Hestetun I, Svendsen MV, Oellingrath IM. Sleep problems and mental health among young Norwegian adolescents. Nord J Psychiatry. 2018;72(8):578-85.

9. Yang KI, Kim JH, Hwangbo Y, Koo DL, Kim D, Hwang KJ, et al. Prevalence of self-perceived snoring and apnea and their association with daytime sleepiness in Korean high school students. J Clin Neurol. 2017;13(3):265-72.

10. Gustafsson ML, Laaksonen C, Salanterä S, Löyttyniemi E, Aromaa M. Changes in the amount of sleep and daytime sleepiness: a follow-up study of schoolchildren from ages 10 to 15 years. Int I Nurs Pract. 2019;25(1):e12689.

11. Hallal PC, Andersen LB, Bull FC, Guthold R, Haskell W, Ekelund U; Lancet Physical Activity Series Working Group. Global physical activity levels: Surveillance progress, pitfalls, and prospects. Lancet. 2012;380(9838):247-57.

12. Tashjian SM, Mullins JL, Galván A. Bedtime autonomy and cellphone use influence sleep duration in adolescents. J Adolesc Health. 2019;64(1):124-30.

13. Amra B, Shahsavari A, Shayan-Moghadam R, Mirheli 0, Moradi-Khaniabadi B, Bazukar M, et al. The association of sleep and late-night cell phone use among adolescents. J Pediatr (Rio J). 2017;93(6):560-7.

14. Tamura H, Nishida T, Tsuji A, Sakakibara H. Association between excessive use of mobile phone and insomnia and depression among Japanese adolescents. Int J Environ Res Public Health. 2017;14(7). pii: E701.

15. Dube N, Khan K, Loehr S, Chu Y, Veugelers P. The use of entertainment and communication technologies before sleep could affect sleep and weight status: A population-based study among children. Int J Behav Nutr Phys Act. 2017;14(1):97.

16. Durusoy R, Hassoy H, Özkurt A, Karababa A0. Mobile phone use, school electromagnetic field levels and related symptoms: a cross-sectional survey among 2150 high school students in Izmir. Environ Health. 2017;16(1):51.

17. Demir YP, Sumer MM. Effects of smartphone overuse on headache, sleep and quality of life in migraine patients. Neurosciences (Riyadh). 2019;24(2):115-21.
18. Lo JC, Lee SM, Lee XK, Sasmita K, Chee NIYN, Tandi J, et al. Sustained benefits of delaying school start time on adolescent sleep and well-being. Sleep. 2018;41(6):zsy052.

19. Temkin DA, Princiotta D, Ryberg R, Lewin DS. Later Start, Longer Sleep: Implications of Middle School Start Times. J Sch Health. 2018;88(5):370-8.

20. Pereira EF, Bernardo MPSL, D'Almeida V, Louzada FM. [Sleep, work, and study: sleep duration in working and non-working students.] Cad Saude Publica. 2011;27(5):975-84.

21. Alves FR, Souza EA, França FLG, Vilar Neto JDO, Bruin VMS, Bruin PFC. Sleep duration and daytime sleepiness in a large sample of Brazilian high school adolescents. Sleep Med. 2020;66:207-15

22. Bray M. Double-shift schooling: design and operation for cost-effectiveness. 3rd ed. Paris: Unesco International Institute for Educational Planning; 2008.

23. Gradisar M, Gardner G, Dohnt $H$. Recent worldwide sleep patterns and problems during adolescence: a review and meta-analysis of age, region, and sleep. Sleep Med. 2011;12(2):110-8.

24. Machado AG, Baptista TJR. 0 corpo nas aulas de Educação Física: concepções e práticas pedagógicas no colégio da polícia militar. Motrivivência. 2019;3(58).

25. Paruthi S, Brooks LJ, D'Ambrosio C, Hall WA, Kotagal S, Lloyd RM, et al. Recommended amount of sleep for pediatric populations: a consensus statement of the American Academy of Sleep Medicine. J Clin Sleep Med. 2016;12(06):785-6.

26. Hirshkowitz M, Whiton K, Albert SM, Alessi C, Bruni 0, DonCarlos L, et al. National Sleep Foundation's sleep time duration recommendations: methodology and results summary. Sleep Health. 2015;1(1):40-43.

27. Bertolazi AN, Fagondes SC, Hoff LS, Pedro VD, Menna Barreto SS, Johns MW. Portugueselanguage version of the Epworth sleepiness scale: validation for use in Brazil. J Bras Pneumol. 2009;35(9):877-83.

28. Anderson B, Storfer-Isser A, Taylor HG, Rosen CL, Redline S. Associations of Executive Function With Sleepiness and Sleep Duration in Adolescents. Pediatrics. 2009;123(4): e701-7.

29. Vilela TS, Bittencourt LRA, Tufik S, Moreira GA. Factors influencing excessive daytime sleepiness in adolescents. J Pediatr (Rio J). 2016;92(2):149-55.

30. Bernardo MPSL, Pereira ÉF, Louzada FM, D’Almeida V. Duração do sono em adolescentes de diferentes níveis socioeconômicos. J Bras Psiquiatr. 2009;58(4):231-7.

31. Wells JCK, Hallal PC, Reichert FF, Menezes AMB, Araújo CLP, Victora CG. Sleep patterns and television viewing in relation to obesity and blood pressure: evidence from an adolescent Brazilian birth cohort. Int J Obes (Lond). 2008:32(7):1042-9.

32. Paiva T, Gaspar T, Matos MG. Mutual relations between sleep deprivation, sleep stealers and risk behaviours in adolescents. Sleep Sci. 2016;9(1):7-13.

33. Sarchiapone M, Mandelli L, Carli V, losue M, Wasserman C, Hadlaczky G, et al. Hours of sleep in adolescents and its association with anxiety, emotional concerns, and suicidal ideation. Sleep Med. 2014;15(2):248-54.

34. Maslowsky J, Ozer EJ. Developmental trends in sleep duration in adolescence and young adulthood: evidence from a national United States sample. J Adolesc Health. 2014;54(6):691-7.

35. Carson V, Tremblay MS, Chaput JP, Chastin SFM. Associations between sleep duration, sedentary time, physical activity, and health indicators among Canadian children and youth using compositional analyses. Appl Physiol Nutr Metab. 2016;41(6):S294-302.

36. Ortega FB, Ruiz JR, Labayen I, Kwak L, Harro J, Oja L, et al. Sleep duration and activity levels in Estonian and Swedish children and adolescents. Eur J Appl Physiol. 2011;111(10):2615-23.

37. Al-Hazzaa H, Musaiger A, Abahussain N, Al-Sobayel H, Qahwaji D. Prevalence of short sleep duration and its association with obesity among adolescents 15- to 19-year olds: A crosssectional study from three major cities in Saudi Arabia. Ann Thorac Med. 2012;7(3):133-9.

38. Paksarian D, Rudolph KE, He JP, Merikangas KR. School Start Time and Adolescent Sleep Patterns: Results from the U.S. National Comorbidity Survey - Adolescent Supplement. Am J Public Health. 2015;105(7):1351-7.

39. Lewin DS, Wang G, Chen YI, Skora E, Hoehn J, Baylor A, et al. Variable school start times and middle school student's sleep health and academic performance. J Adolesc Health. 2017;61(2):205-11.

40. Bacelar AM, Castro CR, Alves R, Araújo P, coords. Dossiê: horários escolares e implicaçōes no sono de adolescentes. e-Coleções FSP/USP. Disponível em: http://colecoes.sibi.usp.br/ fsp/items/show/3362. Acesso em: 15 out. 2019.

41. Schoeni A, Roser K, Röösli M. Symptoms and cognitive functions in adolescents in relation to mobile phone use during night. PLoS One. 2015;10(7):e0133528. 